\title{
Rapid effect of osimertinib re-challenge on brain metastases developing during salvage cytotoxic chemotherapy after osimertinib treatment failure: A case report
}

\author{
AKIMASA SEKINE ${ }^{1}$, HIROAKI SATOH ${ }^{2}$, SATOSHI IKEDA $^{1}$, TSUNEYUKI ODA ${ }^{1}$, \\ KOJI OKUDELA ${ }^{3}$, TAE IWASAWA ${ }^{4}$ and TAKASHI OGURA ${ }^{1}$
}

\begin{abstract}
${ }^{1}$ Department of Respiratory Medicine, Kanagawa Cardiovascular and Respiratory Center, Yokohama, Kanagawa 236-0051;
${ }^{2}$ Department of Internal Medicine, Mito Medical Center, University of Tsukuba, Tsukuba, Ibaraki 310-0015;

${ }^{3}$ Department of Pathology, Graduate School of Medicine, Yokohama City University, Yokohama, Kanagawa 236-0004;

${ }^{4}$ Department of Radiology, Kanagawa Cardiovascular and Respiratory Center, Yokohama, Kanagawa 236-0051, Japan
\end{abstract}

Received October 31, 2018; Accepted February 25, 2019

DOI: $10.3892 / \mathrm{mco} .2019 .1818$

\begin{abstract}
We herein report a case of symptomatic brain metastases (BM) from lung adenocarcinoma in a 73-year-old female patient, which developed during salvage cytotoxic chemotherapy following failure of osimertinib treatment. The patient was proven to have a T790M mutation prior to osimertinib therapy, and achieved a clinical benefit from osimertinib for 3 years until the primary tumor progressed. Although active BM were not detected prior to initiating salvage cytotoxic chemotherapy, the patient developed numbness of the left hand, severe dizziness, and disturbance of behavior and thought after the 3-month course of the salvage cytotoxic chemotherapy. Brain magnetic resonance imaging revealed multiple $\mathrm{BM}$ with severe peritumoral brain edema. To avoid radiation-induced cognitive impairment, osimertinib re-challenge was undertaken. At 2 weeks after osimertinib initiation, the patient's neurological symptoms drastically improved. One month later, radiological evaluation revealed apparent shrinkage of the BM and subsiding brain edema, although the primary lung tumor remained stable. Therefore, osimertinib re-challenge may be a viable treatment option for $\mathrm{BM}$ developing during salvage cytotoxic chemotherapy.
\end{abstract}

\section{Introduction}

Epidermal growth factor-tyrosine kinase inhibitors (EGFR-TKIs) have been proven to be effective for non-small-cell lung cancer (NSCLC) with EGFR mutations. With the advent of

Correspondence to: Dr Akimasa Sekine, Department of Respiratory Medicine, Kanagawa Cardiovascular and Respiratory Center, 6-16-1 Tomiokahigashi, Kanazawa-ku, Yokohama, Kanagawa 236-0051, Japan

E-mail: akimasa.sekine@gmail.com

Key words: osimertinib, gefitinib, brain metastases, re-challenge
EGFR-TKIs, the prognosis of NSCLC has markedly improved, but the incidence of brain metastases (BM) and leptomeningeal metastases (LM) is reportedly increasing, with a reported cumulative incidence of BM of $\sim 46.7 \%$ at 3 years (1). Of note, EGFR-TKIs may also be effective for BM as well as extracranial disease (2); therefore, they are considered as one of the most important therapeutic options. Osimertinib is a third-generation EGFR-TKI, which was designed for NSCLC patients with T790M mutation, and has also been reported to be effective for the treatment of BM (3). However, it remains unclear what treatment strategy would be preferable for BM developing during salvage cytotoxic chemotherapy after osimertinib failure. We herein report a case of a successful osimertinib re-challenge for multiple BM from NSCLC developing during salvage cytotoxic chemotherapy.

\section{Case report}

A 73-year-old female patient was diagnosed with stage IVb lung adenocarcinoma (T1bN2M1b, brain metastases) in April 2013. As there were only two small BM lesions, stereotactic radiosurgery was performed (Fig. 1A). Subsequently, $250 \mathrm{mg}$ gefitinib was administered daily, as the patient was found to harbor an EGFR gene mutation (exon 19 deletion). After 1.5 years of partial response, multiple lung metastases developed. As T790M was detected in the specimen collected by transbronchial lung biopsy, daily treatment with $80 \mathrm{mg}$ osimertinib was initiated, based on the AURA 3 clinical study (AstraZeneca, Cambridge, UK; NCT02151981) (4), resulting in rapid and apparent shrinkage of the primary tumor and multiple lung metastases. Three years later, the primary tumor enlarged, with the cranial lesion remaining stable (Fig. 1B). As one cycle of docetaxel and two cycles of S-1 were ineffective, the patient was administered pemetrexed as fifth-line chemotherapy. Two weeks after the initiation of pemetrexed therapy, however, she developed numbness of the left hand, severe dizziness, and disturbances of behavior and thought, resulting in worsening of the performance status (PS) score to 3. Radiological evaluation revealed the development of 
A

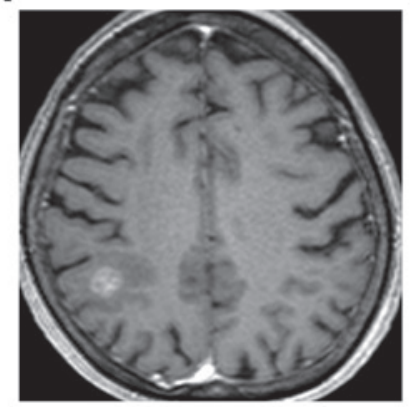

B

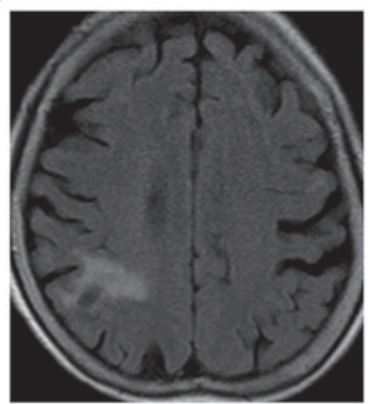

C

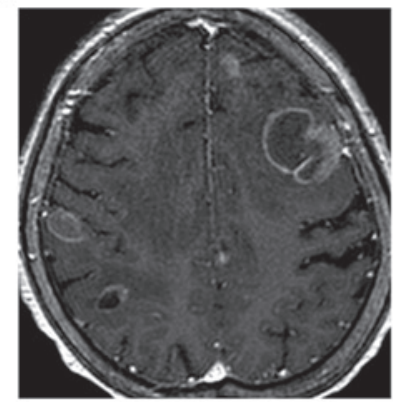

D

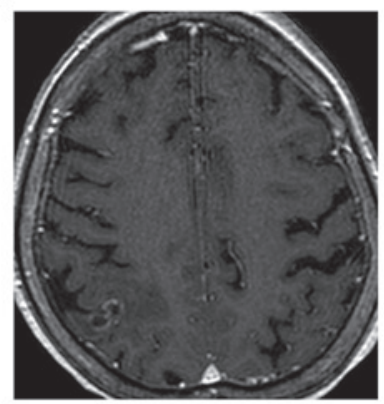

Figure 1. (A) At initial diagnosis, Gadrium-enhanced T1-weighed brain magnetic resonance imaging showing a mass in the right cerebrum with mild peritumoral edema. (B) Only one high-density area was observed (fluid-attenuated resonance imaging). (C) Multiple brain metastases with apparent peritumoral edema developed during salvage cytotoxic chemotherapy. (D) Shrinkage of the multiple brain metastases after initiating osimertinib re-challenge.

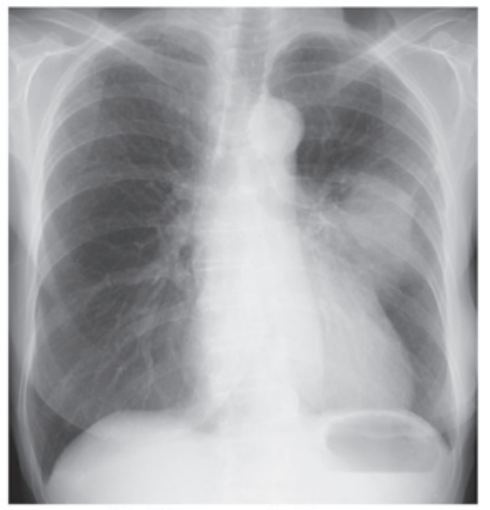

Before re-challenge

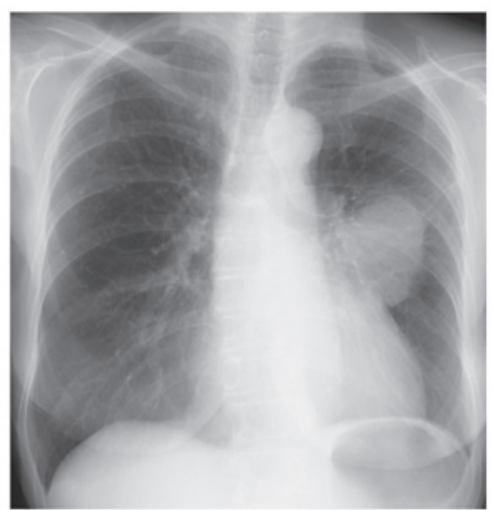

After re-challenge (one month later)

Figure 2. The primary tumor remained stable after initiating osimertinib re-challenge.

multiple BM with severe peritumoral brain edema (Fig. 1C). Whole-brain radiotherapy (WBRT) was excluded due to concerns regarding the exacerbation of the cognitive impairment. Therefore, osimertinib re-challenge therapy (80 $\mathrm{mg} /$ day) was selected. At 2 weeks after treatment initiation, the neurological symptoms drastically improved, with a PS score of 1. One month later, brain magnetic resonance imaging revealed apparent shrinkage of the BM and subsiding brain edema (Fig. 1D), although the primary lung tumor remained stable (Fig. 2). In October 2018, 6 months after initiating osimertinib re-challenge, the patient continued osimertinib treatment and BM remained stable.

\section{Discussion}

The findings of the present case indicate that osimertinib re-challenge may be a viable therapeutic option for BM developing during the course of salvage cytotoxic chemotherapy. There is currently no established optimal therapeutic strategy for BM that develop during salvage cytotoxic chemotherapy following osimertinib failure and disease progression. Radiotherapy, mainly WBRT, may be effective for BM from EGFR-mutated NSCLC, but it is associated with increased risk of neurocognitive impairment (5). In the present case, osimertinib re-challenge was proven to be effective for BM, although there was no change in the primary tumor. Generally, central nervous system progression has been reported as a major concern in NSCLC patients treated with gefitinib, with a prevalence of $35.1 \%$ (6), which is attributable to the penetration rate of the blood-brain barrier (7). With regard to osimertinib, the pre-clinical data indicate favorable penetration into the brain parenchyma (8), which is supported by the marked response of the BM to osimertinib in the present case. Of note, there was a difference in therapeutic efficacy between the BM and the primary lesion; however, as this is beyond the scope of the present case report, this observation is not discussed in detail at present. It is known that there is heterogeneity among T790M-positive cancer cells (9), and it is hypothesized that the difference in therapeutic efficacy in this patient may also be associated with this heterogeneity.

Furthermore, as osimertinib re-challenge acted rapidly on $\mathrm{BM}$, it may be one of the preferable therapies to be considered in the future. Generally, the therapeutic strategy for BM should be decided taking into consideration the activity of extracranial disease and the risk of WBRT-induced cognitive impairment (10). In the present case, osimertinib re-challenge was selected as the patient was elderly and already exhibited signs of cognitive impairment. Due to the rapid and dramatic improvement of the patient's PS within 2 weeks after the initiation of osimertinib re-challenge, there was no need to add radiation to the treatment. Koba et al reported two cases of BM from T790M-positive NSCLC: A rapid response was 
observed 2 weeks later, and WBRT was therefore deemed unnecessary (11). Taking this report together with ours into consideration, osimertinib treatment, even as re-challenge, may exert a rapid and marked effect on BM. Therefore, osimertinib re-challenge may be valuable for WBRT candidates with a concern for potential development of cognitive impairment.

In conclusion, we herein report a case of successful osimertinib re-challenge for BM from lung adenocarcinoma developing during salvage cytotoxic chemotherapy. Although the optimal therapeutic strategy for BM in NSCLC patients previously treated with osimertinib has yet to be determined, the results in the present case suggest that osimertinib re-challenge is a viable treatment option. Accumulation of clinical information in patients with similar treatment status is required to confirm our results.

\section{Acknowledgements}

Not applicable.

\section{Funding}

No funding was received.

\section{Availability of data and materials}

The datasets used and/or analyzed during the present study are available from the corresponding author on reasonable request.

\section{Authors' contributions}

AS and HS participated in the conception and design of the study, analyzed and interpreted the data and wrote the manuscript. AS, SI, TOd and TOg evaluated the patient and participated in the therapy. SI, HS and TOg revised the manuscript for intellectual content. TI and KO evaluated the radiological images or pathological specimens. All authors have read and approved the final draft of the manuscript.

\section{Ethics approval and consent to participate}

Not applicable.

\section{Patient consent for publication}

The patient provided written informed consent for the publication of the case details and any associated images.

\section{Competing interests}

Dr Sekine and Dr Ikeda have received lecture fees from AstraZeneca, Boehringer Ingelheim and Chugai Pharmaceuticals. Dr Ogura has received a lecture fee from Boehringer Ingelheim. The remaining authors have stated that they have no conflicts of interest to disclose.

\section{References}

1. Rangachari D, Yamaguchi N, VanderLaan PA, Folch E, Mahadevan A, Floyd SR, Uhlmann EJ, Wong ET, Dahlberg SE, Huberman MS, et al: Brain metastases in patients with EGFR-mutated or ALK-rearranged non-small-cell lung cancers. Lung Cancer 88: 108-111, 2015.

2. Iuchi T, Shingyoji M, Sakaida T, Hatano K, Nagano O, Itakura M, Kageyama H, Yokoi S, Hasegawa Y, Kawasaki K, et al: Phase II trial of gefitinib alone without radiation therapy for Japanese patients with brain metastases from EGFR-mutant lung adenocarcinoma. Lung Cancer 82: 282-287, 2013.

3. Ahn MJ, Tsai CM, Yang JC, Shepherd FA, Satouchi M, Kim DW, Bazhenova L, Hirashima T, Rukazenkov Y, Cantarini M, et al: AZD9291 activity in patients with EGFR-mutant advanced non-small cell lung cancer (NSCLC) and brain metastases: Data from Phase II studies. Eur J Cancer 51: S625-S626, 2015.

4. Mok TS, Wu YL, Ahn MJ, Garassino MC, Kim HR, Ramalingam SS, Shepherd FA, He Y, Akamatsu H, Theelen WS, et al; AURA3 investigators: Osimertinib or platinum-pemetrexed in EGFR T790M-positive lung cancer. N Engl J Med 376: 629-640, 2017.

5. Tallet AV, Azria D, Barlesi F, Spano JP, Carpentier AF, Gonçalves A and Metellus P: Neurocognitive function impairment after whole brain radiotherapy for brain metastases: Actual assessment. Radiat Oncol 7: 77, 2012.

6. Li MX, He H, Ruan ZH, Zhu YX, Li RQ, He X, Lan BH, Zhang ZM, Liu GD, Xiao HL, et al: Central nervous system progression in advanced non-small cell lung cancer patients with EGFR mutations in response to first-line treatment with two EGFR-TKIs, gefitinib and erlotinib: A comparative study. BMC Cancer 17: 245, 2017.

7. Togashi Y, Masago K, Masuda S, Mizuno T, Fukudo M, Ikemi Y, Sakamori Y, Nagai H, Kim YH, Katsura T, et al: Cerebrospinal fluid concentration of gefitinib and erlotinib in patients with non-small cell lung cancer. Cancer Chemother Pharmacol 70: 399-405, 2012.

8. Ballard P, Yates JW, Yang Z, , Kim DW, Yang JC, Cantarini M, Pickup K, Jordan A, Hickey M, Grist M, et al: Preclinical comparison of osimertinib with other EGFR-TKIs in EGFR-mutant NSCLC brain metastases models, and early evidence of clinical brain metastases activity. Clin Cancer Res 22: 5130-5140, 2016.

9. Hata AN, Niederst MJ, Archibald HL, Gomez-Caraballo M, Siddiqui FM, Mulvey HE, Maruvka YE, Ji F, Bhang HE, Krishnamurthy Radhakrishna V, et al: Tumor cells can follow distinct evolutionary paths to become resistant to epidermal growth factor receptor inhibition. Nat Med 22: 262-269, 2016.

10. Sekine A and Satoh H: Paradigm shift of therapeutic management of brain metastases in EGFR-mutant non-small cell lung cancer in the era of targeted therapy. Med Oncol 34: $121,2017$.

11. Koba T, Kijima T, Takimoto T, Hirata H, Naito Y, Hamaguchi M, Otsuka T, Kuroyama M, Nagatomo I, Takeda Y, et al: Rapid intracranial response to osimertinib, without radiotherapy, in nonsmall cell lung cancer patients harboring the EGFR T790M mutation: Two Case Reports. Medicine (Baltimore) 96: e6087, 2017. 